
МЕДИКАМЕНТОЗНОМУ ЛЕЧЕНИЮ: МЕТОДЫ ОЦЕНКИ И НЕРЕШЕННЫЕ ВОПРОСЫ

Э.Т. Гусейнова, Н.П. Кутишенко, Ю.В. Лукина, С.Н. Толпыгина, В.П. Воронина, С.Ю. Марцевич

ФГБУ «Национальный медицинский исследовательский центр терапии и профилактической медицины» Минздрава России; Россия, 101990 Москва, Петроверигский пер., 10, стр. 3

Контакты: Гусейнова Эльмира Тамерлановна е.guseinova@таil.ru

В статье рассматриваются общие проблемы лечения хронической сердечной недостаточности, среди которых особое внимание уделяется приверженности. Данная проблема - одна из актуальных и труднорешаемых, поскольку непосредственно влияет на эффективность лечения и исход заболевания. Рассмотрена приверженность соблюдению клинических рекомендаций со стороны врачей и назначаемой терапии - со стороны пациентов. Описаны некоторые причины, объясняющие недостаточную приверженность лечению (пол, возраст, учреждение, в котором пациент наблюдается и др.).

Отяжеляющее обстоятельство в лечении хронической сердечной недостаточности - коморбидность. Наличие сопутствующих заболеваний увеличивает количество принимаемых препаратов, что усугубляет проблему приверженности медикаментозной терапии. Отмечено, что идеальной моделью изучения приверженности в клинической практике остается медицинский регистр. В статье рассмотрены основные российские регистры пациентов с хронической сердечной недостаточностью. Обозначено, что ни в одном из них полноценная оценка приверженности пациентов терапии не проводилась. В наиболее крупных зарубежных регистрах хронической сердечной недостаточности оценке приверженности уделяется также недостаточно внимания.

В отдельных публикациях дана оценка приверженности врачей назначению рекомендованной терапии. Терапия должна быть качественной и соответствовать действующим клиническим рекомендациям, что свидетельствует о достаточной приверженности врача медикаментозному лечению. Отдельно описаны клинические исследования, в том числе рандомизированные, специально изучающие различные аспекты проблемы приверженности и ее влияние на течение болезни. Также внимание уделено методам оценки приверженности в этих исследованиях. Отмечено, что нет «золотого стандарта» ее оценки, а существующие методы (такие как чипы, определение концентрации препарата в крови, подсчет рецептурных бланков и др.) неприемлемы для реальной клинической практики. Это говорит о необходимости и перспективах дальнейшей работы по оценке приверженности терапии у больных с хронической сердечной недостаточностью.

Ключевые слова: регистр, приверженность, коморбидность, хроническая сердечная недостаточность, методы оценки приверженности, опросник, клинические исследования, лекарственная терапия, изучение приверженности терапии, клинические рекомендации

Для цитирования: Гусейнова Э.Т., Кутишенко Н.П., Лукина Ю.В. и др. Проблема хронической сердечной недостаточности и приверженности медикаментозному лечению: методы оценки и нерешенные вопросы. Клиницист 2020;14(3-4)-К627.

DOI: $10.17650 / 1818-8338-2020-14-3-4-K 627$

(cc) BY

\title{
CHRONIC HEART FAILURE AND ADHERENCE TO MEDICATION: METHODS FOR ASSESSING ADHERENCE TO THERAPY AND UNRESOLVED ISSUES
}

\author{
E.T. Guseinova, N.P. Kutishenko, Yu.V. Lukina, S.N. Tolpygina, V.P. Voronina, S. Yu. Martsevich \\ National Medical Research Center for Therapy and Preventive Medicine of the Ministry of Health of Russia; \\ 10 Build 3 Petroverigskiy Pereulok, Moscow 101990, Russia
}

The review of the literature examines the General problems of treatment of chronic heart failure, among which special attention is paid to the problem of adherence. This problem remains one of the most urgent and difficult to solve in medicine, because it directly affects the effectiveness of treatment and the outcome of the disease. The article considers the commitment of both doctors to comply with clinical recommendations and patients to prescribed therapy. Some of the reasons that explain the lack of adherence to treatment (such as gender, age, institution where the patient is observed, and others) are described. It is also described that comorbidity is an aggravating factor in the treatment of chronic heart failure. It is described that comorbidity is an aggravating factor in the treatment of chronic heart failure. The presence of concomitant diseases increases the number of medications taken, which exacerbates the problem of adherence to medication therapy. It is noted that medical registry is the ideal model for studying adherence in clinical practice. The main Russian registers included of patients with chronic heart failure are considered. It is noted that in none of them a full assessment of patients' adherence to therapy was carried out. In the largest foreign chronic heart failure registers, the assessment of commitment is also given insufficient attention. Separate clinical trials 
are described, including randomized trials, specifically studying various aspects of the problem of adherence and its impact on the course of the disease. Special attention is paid to the methods of assessing adherence in these studies. It is noted that there is no "gold standard" for its evaluation, and existing methods (such as Chips, determining the concentration of the drug in the blood, counting prescription forms and others) are unacceptable for real clinical practice. This indicates the need and prospects for further work on assessing adherence to therapy in patients with chronic heart failure.

Key words: registry, adherence, comorbidity, chronic heart failure, methods for assessing treatment, questionnaire, clinical research, drug therapy, study of adherence to therapy, guidelines

For citation: Guseinova E.T., Kutishenko N.P., Lukina Yu.V. et al. Chronic heart failure and adherence to medication: methods for assessing adherence to therapy and unresolved issues. Klinitsist = The Clinician 2020;14(3-4)-K627. (In Russ.).

\section{Введение}

Хроническая сердечная недостаточность (ХCH) одна из основных причин смертности от сердечно-сосудистых заболеваний. По данным Фремингенского исследования, средняя 5-летняя смертность во всей популяции больных с ХСН остается высокой и составляет 62-65 \% для мужчин и 42-47 \% для женщин. Как правило, ХСН - финал практически всех заболеваний сердечно-сосудистой системы или ее поражения при других патологических процессах. Сложности в диагностике, недостаточная эффективность медикаментозной терапии, высокая частота регоспитализаций, неблагоприятный прогноз и финансовые затраты определяют медицинскую и социально-экономическую значимость заболевания во всем мире.

Данные рандомизированных клинических исследований (РКИ), проводившихся с применением ингибиторов ангиотензинпревращающего фермента (иАПФ), $\beta$-адреноблокаторов ( $\beta$-АБ), антагонистов минералокортикоидов, свидетельствуют о том, что продолжительность жизни пациентов с ХСН может быть увеличена. Результаты этих исследований нашли отражение в современных клинических рекомендациях [1].

\section{общие проблемы лечения хронической}

\section{сердечной недостаточности}

Отяжеляющее обстоятельство в лечении больных с ХCH - коморбидность. Она ухудшает прогноз, повышает риск повторных госпитализаций, увеличивает степень полипрагмазии. Сама ХСН, согласно клиническим рекомендациям, требует, как правило, назначения нескольких препаратов, таких как иАПФ, антагонисты минералокортикоидов, $\beta$-АБ. Кроме того, нередко необходима симптоматическая терапия: назначение диуретиков, сердечных гликозидов. Не следует забывать, что лечение сопутствующих заболеваний (сахарного диабета, хронической обструктивной болезни легких, хронической болезни почек), которые часто встречаются у пациентов с ХСН, увеличивает количество принимаемых препаратов.

По данным европейского исследования (European Heart Failure Pilot Survey), у 74 \% больных с ХCH наблюдается не менее одного сопутствующего заболевания, наиболее часто встречаются хроническая болезнь почек (41 \%), анемия (29 \%) и сахарный диабет (29 \%) [2]. При исследовании когнитивных функций у каждого второго больного с ХСН наблюдаются преддементные когнитивные нарушения, у каждого пятого - деменция легкой степени выраженности. По данным литературы, для больных с ХСН характерны нарушения памяти и психомоторные расстройства [3].

Относительный риск смерти в течение 12 мес в группе больных с высокой коморбидностью (индекс коморбидности Чарлсона $>6$ баллов) в 1,68 раза (95\% доверительный интервал 1,35-2,09) выше риска смерти больных с низкой коморбидностью. Коморбидность приводит к увеличению числа и длительности госпитализаций как по всем причинам, так и в связи с обострением сердечно-сосудистых заболеваний, включая декомпенсацию ХСН. При ХСН с высокой коморбидностью (индекс коморбидности Чарлсона $>6$ баллов) среднегодовая длительность госпитализаций по всем причинам больше на 5 дней, а в связи с обострением сердечно-сосудистых заболеваний - на 3 дня [4].

Из этого следует, что больной с ХСН должен получать препараты для лечения как основного заболевания, так и сопутствующей патологии. А прием большого количества препаратов всегда сопряжен с проблемой приверженности рекомендованной терапии.

\section{Проблема приверженности}

Согласно определению, предложенному экспертами Всемирной организации здравоохранения, приверженность лечению - это «степень соответствия поведения пациента рекомендациям врача в отношении регулярности приема лекарственных препаратов, его дозы и интервала между приемами, а повышение эффективности мероприятий, направленных на улучшение приверженности лечению, может иметь гораздо большее влияние на здоровье населения, чем усовершенствование каких-либо отдельных медицинских процедур». Приверженность - это сложный поведенческий процесс, определяемый многими взаимодействующими факторами, связанными с пациентом, врачом, организацией системы здравоохранения, лекарственной терапией, особенностями заболевания, а также социально-экономическими факторами [5]. 
Большинство специалистов, изучающих вопросы приверженности, отмечают отсутствие заметного прогресса в данной проблеме, неадекватные методы ее оценки, низкую эффективность большинства предлагаемых методов по улучшению приверженности. Следует отметить, что на приверженность лечению влияют частота посещений пациентом лечебного учреждения и наследственность, связанная с сердечнососудистым заболеванием. Так, при анализе результатов регистра «ПРОФИЛЬ» (регистр отдела профилактической фармакотерапии ФГБУ «НМИЦ ПМ» - 177 больных, включенных в регистр) выявлено, что пациенты без отягощенной наследственности менее привержены лечению $(p=0,019)$. Анализируя результаты этого регистра, мы узнали, что более приверженными врачебным рекомендациям оказались те пациенты, которые бывают на визите у врача не реже 1 раза в 2 года $(p=0,014)[6,7]$.

Недостаточная приверженность снижает качество терапии и ее эффективность. Одна из проблем приверженности - отсутствие «золотого стандарта» ее оценки. При анализе контролируемых исследований было выяснено, что в развитых странах приверженность пациентов лечению хронических заболеваний составляет в среднем лишь $50 \%$. Это серьезная проблема для здравоохранения, поскольку эффективность лечения и продолжительность ремиссии определяются в первую очередь приверженностью пациентов назначениям врача [8]. В вопросе приверженности большое значение имеет качество назначаемой терапии, т. е. соответствие ее действующим клиническим рекомендациям. Понятно, что высокая приверженность хороша лишь тогда, когда медикаментозная терапия подобрана качественно. Приверженность терапии off-label («терапия вне инструкции») может ухудшить прогноз и отрицательно сказаться на результатах лечения [9]. Все это позволяет говорить о приверженности врача и пациента как двух неразрывно связанных звеньев одной цепи.

Несмотря на большое количество исследований, посвященных изучению приверженности при различных заболеваниях, относительно больных с ХСН эта проблема изучена недостаточно. Имеются отдельные работы по изучению приверженности в рамках клинических исследований. В этих исследованиях используются чипы, определяют концентрацию препарата в крови и т.д., однако эти методы неприемлемы в рамках повседневной клинической практики. Подсчет рецептурных бланков, широко применяемый при анализе регистров и баз данных, также не отражает картину, поскольку тот факт, что препарат выписан и получен, не означает, что пациент его действительно принимает.

Достаточно надежная модель для изучения и оценки приверженности - клинический регистр. Он всегда подразумевает контакт врача с больным и, соответственно, более наглядно отражает проблему.
Цель данной статьи - изучение состояния проблемы приверженности лечению больных с ХCH в современной доступной литературе.

\section{Регистры и их роль в клинической практике}

\section{Российские регистры}

В Российской Федерации существует ряд регистров и эпидемиологических исследований пациентов с ХСН. Цели этих регистров были разные, оценка приверженности терапии проводилась не в каждом (табл. 1).

Регистр RUS-HFR (Russian hoSpital Heart Failure Registry) ставил целью изучение этиологии XCH, причин госпитализации больных и соответствия их терапии международным рекомендациям. Регистр проводили на базе 3 центров, соответственно, результаты были различны. Так, в ФГБУ «Национальный медицинский исследовательский центр им. В.А. Алмазова» Минздрава России основными причинами госпитализации были плановые хирургические вмешательства при заболеваниях сердечно-сосудистой системы. В ГБОУ ВПО «Оренбургская государственная медицинская академия» Минздрава России большинство больных с ХСН были госпитализированы по поводу нестабильной стенокардии $(57,1 \%)$ и нарушений ритма сердца $(22,2 \%)$. Оценка приверженности терапии у этих больных не описана [10].

В Павловском регистре ХСН проводилась оценка выживаемости, течения ХСН у пациентов с разными фенотипами, оценивалась терапия, назначенная врачами, т. е. оценивалась приверженность последних. Выявлено, что практически половина пациентов $(44,1 \%)$ до декомпенсации ХСН не получали иАПФ, 48,2 \% не получали $\beta$-АБ, что связано с недостаточной приверженностью врачей соблюдению клинических рекомендаций [15].

В «ОРАКУЛ-РФ» проводилась оценка летальности и частоты регоспитализаций на 30, 90, 180, 360-й день наблюдения, а также распространенности сопутствующих заболеваний у больных с ХСН. Авторы предлагают формировать фенотипы пациентов с XCH на основании сопутствующей патологии. Вопросы приверженности пациентов в данных регистрах также не поднимались или не опубликованы [12].

В регистрах РЕКВАЗА и ЛИС-2, вКлючивших определенную часть больных с ХСН, проводилась оценка приверженности терапии с помощью опросника Мориски-Грина, который не оценивает приверженность лечению конкретными препаратами, а определяет лишь готовность пациента выполнять рекомендации врача. В РЕКВАЗА было выявлено, что приверженность лечению выше у пациентов с постинфарктным кардиосклерозом (4 балла) по сравнению с больными без него [16] (табл. 2).

Попытка оценить приверженность терапии на амбулаторном этапе выполнена в Красноярске. В исследование были включены 150 специально отобранных 
Таблица 1. Российские регистры и эпидемиологические исследования, в которые включались пациенты с хронической сердечной недостаточностью Table 1. Russian registries and epidemiological studies, which included patients with chronic heart failure

\begin{tabular}{|c|c|c|c|c|}
\hline $\begin{array}{c}\text { Регистр/крупное } \\
\text { исследование } \\
\text { Registry/large-scale study }\end{array}$ & $\begin{array}{l}\text { Основные характеристики } \\
\text { больных } \\
\text { Main characteristics of patients }\end{array}$ & $\begin{array}{l}\text { Число больных } \\
\text { Number of patients }\end{array}$ & $\begin{array}{c}\text { Способ } \\
\text { включения } \\
\text { Inclusion } \\
\text { in the study }\end{array}$ & $\begin{array}{c}\text { Проводилась ли оценка } \\
\text { приверженности } \\
\text { Adherence to therapy }\end{array}$ \\
\hline $\begin{array}{l}\text { RUS-HFR [10]. } \\
\text { C } 2012 \text { г. по июнь } 2013 \text { г. } \\
\text { From } 2012 \text { to June } 2013\end{array}$ & $\begin{array}{c}\text { АГ, ИБС, ФП, } \\
\text { кардиомиопатии } \\
\mathrm{AH}, \mathrm{CHD}, \mathrm{AF} \text {, cardiomyopathy }\end{array}$ & 251 & $\begin{array}{l}\text { Последо- } \\
\text { вательно } \\
\text { Consecutive }\end{array}$ & $\begin{array}{c}\text { Не проводилась } \\
\text { или не опубликована } \\
\text { Not assessed or not } \\
\text { published }\end{array}$ \\
\hline $\begin{array}{l}\text { Регистр в Нижнем } \\
\text { Новгороде [11]. С } 2014 \text { г., } \\
\text { возможно, исследование } \\
\text { продолжается } \\
\text { Register in Nizhny Novgorod } \\
\text { [11]. Since 2014, possibly } \\
\text { continues }\end{array}$ & $\begin{array}{c}\text { Острая декомпенсация } \\
\text { XCH } \\
\text { Acute decompensated HF }\end{array}$ & 648 & $\begin{array}{l}\text { Последо- } \\
\text { вательно } \\
\text { Consecutive }\end{array}$ & $\begin{array}{c}\text { Прямая оценка } \\
\text { не проводилась } \\
\text { Not assessed directly }\end{array}$ \\
\hline $\begin{array}{l}\text { «ОРАКУЛ-РФ» [12] } \\
\text { ORACUL-RF [12] }\end{array}$ & $\begin{array}{c}\text { Декомпенсация XCH } \\
\text { Decompensated HF }\end{array}$ & 2450 & $\begin{array}{c}\text { Последо- } \\
\text { вательно } \\
\text { Consecutive }\end{array}$ & $\begin{array}{l}\text { Hе проводилась } \\
\text { Not assessed }\end{array}$ \\
\hline $\begin{array}{l}\text { РИФ-ХСН [13] } \\
\text { RIF-CHD [13] }\end{array}$ & $\begin{array}{l}\text { ХСН в сочетании с ФП } \\
\text { CHF in combination with AF }\end{array}$ & 1003 & $\begin{array}{c}\text { Последователь- } \\
\text { но } \\
\text { Consecutive }\end{array}$ & $\begin{array}{c}\text { Приверженность } \\
\text { пациентов не оцени- } \\
\text { валась, проводилась } \\
\text { оценка приверженно- } \\
\text { сти врачей назначе- } \\
\text { нию адекватной, } \\
\text { рекомендованной } \\
\text { терапии } \\
\text { Patient adherence was not } \\
\text { assessed; physician } \\
\text { adherence to adequate, } \\
\text { recommended therapy was } \\
\text { assessed }\end{array}$ \\
\hline $\begin{array}{l}\text { «НПОХА-О-ХСН» [14] } \\
\text { ЕРОНА-О-СНD [14] }\end{array}$ & $\begin{array}{l}\text { ХСН, АГ, ИБС } \\
\text { СНF, АG, СНD }\end{array}$ & $\begin{array}{c}6884 \text { из скрининг- } \\
\text { форм } 17824 \text { больных } \\
\text { (22 региона России) } \\
6884 \text { of } 17824 \text { screening } \\
\text { forms (22 regions of the } \\
\text { Russia) }\end{array}$ & $\begin{array}{l}\text { Выборочно } \\
\text { Randomly }\end{array}$ & $\begin{array}{c}\text { Прямая оценка } \\
\text { не проводилась } \\
\text { Not assessed directly }\end{array}$ \\
\hline $\begin{array}{l}\text { Павловский } \\
\text { регистр ХСН [15] } \\
\text { Pavlovsky registry of CHF [15] }\end{array}$ & $\begin{array}{c}\text { Подтвержденная XСH } \\
\text { III-IV ФК } \\
\text { Confirmed CHF III-IV AF }\end{array}$ & 1001 & $\begin{array}{l}\text { Последо- } \\
\text { вательно } \\
\text { Consecutive }\end{array}$ & $\begin{array}{l}\text { Hе проводилась } \\
\text { Not assessed }\end{array}$ \\
\hline \multicolumn{5}{|c|}{$\begin{array}{l}\text { Примечание. Здесь и в табл. 2: АГ- артериальная гипертензия; ИБС- -ишемическая болезнь сердиа; ФП - фибрилляция } \\
\text { предсердий; ХСН - хроническая сердечная недостаточность. }\end{array}$} \\
\hline
\end{tabular}

амбулаторных больных с ХСН: 85 \% имели изолированное нарушение диастолической функции, у 60,7 \% в основе заболевания лежало сочетание ишемической болезни сердца и артериальной гипертензии, у 24,7\% больных ХСН стала следствием артериальной гипертензии, у $12 \%$ пациентов причиной $\mathrm{XCH}$ был порок сердца. Остальные 2,6 \% больных имели другие причины ХСН. Для оценки приверженности пациентов через год применяли также опросник Мориски-Грина. Проанализировав полученные данные, авторы выявили: 56 (37,3\%) пациентов периодически забывают принять препараты; 66 (44 \%) человек указали, что невнимательно относятся ко времени приема препарата; 45 (30 \%) пациентов отметили, что прекращают прием препаратов, когда чувствуют себя хорошо; 27 (18\%) больных прекращают прием препаратов, когда чувствуют себя лучше. Таким образом, большая часть амбулаторных больных с ХСН оказались не привержены медикаментозной терапии. Что касается оценки приверженности врачей, то при анонимном анкетировании выявлено, что большинство (56 \%) амбулаторных врачей имели ту или иную фазу эмоционального выгорания, что могло отрицательно сказываться на качестве лечения больных с ХCH [18]. 
Таблица 2. Российские регистры, посвященные сердечно-сосудистым заболеваниям, в которых присутствовала часть больных с хронической сердечной недостаточностью

Table 2. Russian registries on cardiovascular diseases, which included patients with chronic heart failure

\begin{tabular}{|c|c|c|c|c|}
\hline $\begin{array}{l}\text { Регистр } \\
\text { Registry }\end{array}$ & $\begin{array}{c}\text { Основные } \\
\text { характеристики больных } \\
\text { Main characteristics } \\
\text { of patients }\end{array}$ & $\begin{array}{l}\text { Число больных } \\
\text { с ХСН } \\
\text { Number of patients }\end{array}$ & $\begin{array}{c}\text { Способ } \\
\text { включения } \\
\text { Inclusion in the study }\end{array}$ & $\begin{array}{l}\text { Проводилась ли оценка } \\
\text { приверженности } \\
\text { Adherence to therapy }\end{array}$ \\
\hline $\begin{array}{l}\text { РЕКВАЗА-КЛИ- } \\
\text { НИКА [16] } \\
\text { C 01.04.2013 г. } \\
\text { REKVAZA-CLINIC } \\
\text { [16]. Since } 01.04 .2013\end{array}$ & $\begin{array}{c}\text { ФП в сочетании с АГ, } \\
\text { ИБС, ХСН } \\
\text { AF in combination with } \mathrm{AH}, \\
\text { CHD, CHF }\end{array}$ & $\begin{array}{c}\text { Включены } 285 \\
\text { из } 3696 \\
\text { Included } 285 \\
\text { of } 3696\end{array}$ & $\begin{array}{l}\text { Последо- } \\
\text { вательно } \\
\text { Consecutive }\end{array}$ & $\begin{array}{c}\text { Проводилась оценка приверженности } \\
\text { врачей назначению адекватной } \\
\text { рекомендованной терапии } \\
\text { Physician adherence to adequate, recommended } \\
\text { therapy was assessed }\end{array}$ \\
\hline $\begin{array}{l}\text { PEKBA3A [17] } \\
\text { REKVAZA [17] }\end{array}$ & $\begin{array}{l}\text { ХСН, ИБС и АГ } \\
\text { CHF, СНD and АН }\end{array}$ & 2304 & $\begin{array}{l}\text { Последо- } \\
\text { вательно } \\
\text { Consecutive }\end{array}$ & $\begin{array}{c}\text { Проводилась по опроснику } \\
\text { Мориски-Грина } \\
\text { Assessed using Morisk - Green questionnaire }\end{array}$ \\
\hline $\begin{array}{l}\text { ЛИС-2 } \\
\text { LIS-2 }\end{array}$ & $\begin{array}{c}\text { Мозговой инсульт, } \\
\text { сердечно-сосудистые } \\
\text { и эндокринные заболе- } \\
\text { вания } \\
\text { Cerebral stroke, } \\
\text { cardiovascular and endocrine } \\
\text { diseases }\end{array}$ & 47 & $\begin{array}{l}\text { Последо- } \\
\text { вательно } \\
\text { Consecutive }\end{array}$ & $\begin{array}{c}\text { Проводилась оценка приверженности } \\
\text { пациентов с мозговым инсультом } \\
\text { (включены были и пациенты с ХСН) } \\
\text { по опроснику Мориски-Грина } \\
\text { Adherence of patients with cerebral stroke was } \\
\text { assessed (patients with CHF were also included) } \\
\text { using Morisky-Green questionnaire }\end{array}$ \\
\hline
\end{tabular}

Отдельные работы, посвященные оценке приверженности, проведены в Оренбурге, но не в рамках регистра. В терапевтическом стационаре и поликлинике областной клинической больницы № 2 были обследованы 120 произвольно выбранных больных для оценки приверженности. Из них 100 человек были включены в «группу воздействия». В данной группе проводилась коррекция низкой приверженности с помощью индивидуальных бесед, после чего было проведено сравнение приверженности в разных группах. Полученные данные информативны для оценки отношения пациента к назначению врача и выявления отклика, но первоначальную приверженность терапии и факторы, влияющие на нее, мы не получим. Разработанная анкета для оценки приверженности включает 30 вопросов и сложна для использования в рутинной практике [19].

В повседневной клинической практике предлагается опросник, разработанный в научно-диспансерном отделе Института клинической кардиологии им. А.Л. Мясникова ФГБУ «Российский кардиологический научно-производственный комплекс» Минздрава России (Агеев Ф.Т. и др., 2008, 2013). Но он также не адаптирован для больных с ХСН и, вероятно, более удобен для больных с гипертонической болезнью.

\section{Зарубежные регистры}

Многочисленные зарубежные регистры изучали в основном течение заболевания, назначение терапии и ее влияние на частоту госпитализаций. В отдельных публикациях описана оценка приверженности врачей назначению рекомендованной терапии. Данные о приверженности пациентов лечению оценивались не вез- де и способами, недоступными для реальной клинической практики (табл. 3).

ADHERE (Acute Decompensated Heart Failure National Registry) - один из самых крупных многоцентровых регистров, насчитывающий более 100 тыс. пациентов, госпитализированных по поводу острой декомпенсации сердечной недостаточности. При анализе публикаций по данным этого регистра выявлено, что на прогноз влияло отделение, куда был госпитализирован пациент. Кроме того, проведен анализ клинической картины заболевания, рекомендованной терапии и сопутствующих нозологий, определяющих коморбидность. Была оценена адекватность терапии действующим на тот момент клиническим рекомендациям. Данные об оценке приверженности пациентов не опубликованы, но о приверженности врачей можно судить по назначению рекомендованной терапии [20, 21].

Отличительной особенностью регистра ОРТIMIZE-HF (Organized Program to Indicate Life Saving Treatment in Hospitalized Patients with Heart Failure) была попытка улучшения медицинской помощи пациентам с ХCH. В нем также проводилось обучение врачей действующим рекомендациям, а госпитализированные пациенты были призваны следовать им. В данном регистре оценивалась частота назначения рекомендованной терапии пациентам с ХCH, т. е. рассматривался вопрос приверженности врачей. В ходе программы увеличилось число назначений лечащими врачами иАПФ и $\beta$-АБ, улучшающих прогноз данной когорты больных. На примере $\beta$-АБ показана частота одногодичной летальности при назначении препаратов одной группы с доказанной эффективностью и без нее при соблюдении регулярности приема препаратов. По результатам 
Таблица 3. Зарубежные регистры и крупные исследования больных с хронической сердечной недостаточностью Table 3. Foreign registries and large-scale studies of patients with chronic heart failure

\begin{tabular}{|c|c|c|c|c|}
\hline $\begin{array}{l}\text { Регистр/крупное } \\
\text { исследование } \\
\text { Registry/large-scale study }\end{array}$ & $\begin{array}{l}\text { Число } \\
\text { больных } \\
\text { Number } \\
\text { of patients }\end{array}$ & $\begin{array}{l}\text { Способ } \\
\text { включения } \\
\text { Inclusion } \\
\text { in the study }\end{array}$ & $\begin{array}{c}\text { Проводилась ли оценка } \\
\text { приверженности } \\
\text { Adherence to therapy }\end{array}$ & $\begin{array}{l}\text { Оценка исходов } \\
\text { Outcome assessment }\end{array}$ \\
\hline $\begin{array}{l}\text { ADHERE } \\
\text { Многоцентровой } \\
\text { регистр }[20,21] \\
\text { Multicenter registry }[20,21]\end{array}$ & $\begin{array}{l}\text { Более } \\
107 \text { тыс. } \\
\text { More than } \\
107 \text { thousand }\end{array}$ & $\begin{array}{l}\text { Последо } \\
\text { вательно } \\
\text { Consecutive }\end{array}$ & $\begin{array}{l}\text { Не проводилась } \\
\text { Consecutive }\end{array}$ & $\begin{array}{c}\text { Оценка длительности госпитализации } \\
\text { и частоты летальных исходов } \\
\text { Length of hospital stay and frequency } \\
\text { of deaths were assessed }\end{array}$ \\
\hline $\begin{array}{l}\text { OPTIMIZE-HF } \\
\text { Многоцентровой } \\
\text { регистр [22, 23] } \\
\text { Multicenter registry }[22,23]\end{array}$ & 48612 & $\begin{array}{l}\text { Последо- } \\
\text { вательно } \\
\text { Consecutive }\end{array}$ & $\begin{array}{c}\text { Не проводилась } \\
\text { Not assessed }\end{array}$ & $\begin{array}{c}\text { Определена } 30-, 60-, 90 \text {-дневная и годичная } \\
\text { госпитальная летальность. Проведена } \\
\text { оценка выполнения врачами рекомендаций } \\
\text { Determined } 30-, 60-, 90 \text {-day and one-year hospital } \\
\text { mortality. Doctors' compliance with } \\
\text { recommendations was assessed }\end{array}$ \\
\hline $\begin{array}{l}\text { IMPАCT-HF } \\
\text { Одноцентровой } \\
\text { регистр [24, 25] } \\
\text { Single center registry }[24,25]\end{array}$ & 567 & $\begin{array}{l}\text { Последо- } \\
\text { вательно } \\
\text { Consecutive }\end{array}$ & $\begin{array}{l}\text { Не проводилась } \\
\text { Not assessed }\end{array}$ & $\begin{array}{c}\text { Оценка 60-дневной смертности } \\
\text { и выживаемости на фоне терапии } \\
\beta \text {-адреноблокаторами } \\
\text { 60-day mortality and survival with beta-blocker } \\
\text { therapy were assessed }\end{array}$ \\
\hline $\begin{array}{l}\text { QUALIFY } \\
\text { Многоцентровой } \\
\text { регистр [26] } \\
\text { Multicenter registry [26] }\end{array}$ & 6118 & $\begin{array}{l}\text { Последо- } \\
\text { вательно } \\
\text { Consecutive }\end{array}$ & $\begin{array}{c}\text { Оценка привержен- } \\
\text { ности врачей назначе- } \\
\text { нию рекомендован- } \\
\text { ной терапии } \\
\text { Physician adherence to } \\
\text { recommended therapy was } \\
\text { assessed }\end{array}$ & $\begin{array}{c}\text { Оценка летальности и частоты повторных } \\
\text { госпитализаций } \\
\text { Mortality and readmission rate were assessed }\end{array}$ \\
\hline $\begin{array}{l}\text { СОАСН-2 } \\
\text { PКИ [27] } \\
\text { RCТ [27] }\end{array}$ & 189 & $\begin{array}{l}\text { Нет данных } \\
\text { Data not } \\
\text { available }\end{array}$ & $\begin{array}{c}\text { Оценка привержен- } \\
\text { ности врачей (GAI-3), } \\
\text { оценка приверженно- } \\
\text { сти пациентов } \\
\text { по методу MPR } \\
\text { Physician adherence } \\
\text { (GAI-3), patient } \\
\text { adherence using MPR } \\
\text { method }\end{array}$ & $\begin{array}{c}\text { Оценка летальности и повторных } \\
\text { госпитализаций по поводу дестабилизации } \\
\text { ХСН и других кардиальных событий } \\
\text { за год наблюдений } \\
\text { Mortality and re-hospitalizations for destabilization } \\
\text { of CHF and other cardiac events during the year } \\
\text { of observation }\end{array}$ \\
\hline $\begin{array}{l}\text { HART } \\
\text { PKИ [28] } \\
\text { RCT [28] }\end{array}$ & 902 & $\begin{array}{l}\text { Добро- } \\
\text { вольно } \\
\text { Voluntarily }\end{array}$ & $\begin{array}{l}\text { Оценка привержен- } \\
\text { ности врачей и паци- } \\
\text { ентов (MEMS) } \\
\text { Physician and patient } \\
\text { adherence assessed } \\
\text { (MEMS) }\end{array}$ & $\begin{array}{c}\text { Наблюдение в течение } 12 \text { мес } \\
\text { и определение факторов, влияющих } \\
\text { на приверженность } \\
\text { Follow-up for } 12 \text { months and identification } \\
\text { of factors affecting adherence }\end{array}$ \\
\hline $\begin{array}{l}\text { ESC-HF Pilot } \\
\text { Многоцентровое } \\
\text { наблюдательное } \\
\text { исследование [29] } \\
\text { Multicenter observational } \\
\text { study [29] }\end{array}$ & 51189 & $\begin{array}{l}\text { Нет данных } \\
\text { Data not } \\
\text { available }\end{array}$ & $\begin{array}{c}\text { Не проводилась } \\
\text { Not assessed }\end{array}$ & $\begin{array}{c}\text { Оценка летальности на фоне ОСН и ХСН, } \\
\text { госпитализаций в связи } \\
\text { с дестабилизацией ХСН } \\
\text { Mortality at the background of AHF and CHF, } \\
\text { hospitalizations due to destabilization of CHF } \\
\text { were assessed }\end{array}$ \\
\hline $\begin{array}{l}\text { Euro Heart Failure Survey } \\
\text { Многоцентровое } \\
\text { исследование [30] } \\
\text { Multicenter study [30] }\end{array}$ & $\begin{array}{l}\text { Более } \\
11 \text { тыс. } \\
\text { More than } \\
11 \text { thousand }\end{array}$ & $\begin{array}{l}\text { Последо- } \\
\text { вательно } \\
\text { Consecutive }\end{array}$ & $\begin{array}{l}\text { Не проводилась } \\
\text { Not assessed }\end{array}$ & $\begin{array}{c}\text { Оценка госпитальной смертности } \\
\text { и летальности после выписки из стационара } \\
\text { Assessment of in-hospital mortality and mortality } \\
\text { after discharge from hospital }\end{array}$ \\
\hline $\begin{array}{l}\text { IN-CHF } \\
\text { Многоцентровой } \\
\text { регистр [31] } \\
\text { Multicenter registry [31] }\end{array}$ & 3327 & $\begin{array}{l}\text { Последо- } \\
\text { вательно } \\
\text { Consecutive }\end{array}$ & $\begin{array}{l}\text { Не проводилась } \\
\text { Not assessed }\end{array}$ & $\begin{array}{c}\text { Оценивались смертность и частота повтор- } \\
\text { ных госпитализаций пациентов с ХСН } \\
\text { Assessment of mortality and readmission rate } \\
\text { of patients with CHF }\end{array}$ \\
\hline
\end{tabular}

Примечание. РКИ - рандомизированное клиническое исследование; ОСН - острая сердечная недостаточность; ХСН - хроническая сердечная недостаточность.

Note. RCT - randomized clinical trial; $A H F$ - acute heart failure; $C H F$ - chronic heart failure. 
исследования уровень госпитальной смертности снизился с 4,1 до 2,5 \% [22]. В противоположность данным крупных РКИ в результатах данного исследования говорится, что начало терапии $\beta$-АБ сопровождается увеличением частоты госпитализаций пациентов с ХCH с низкой фракцией выброса. Авторы объясняют это отрицательным инотропным эффектом группы и, возможно, недостаточной приверженностью пациентов терапии (оценка приверженности лечению не проводилась). Во время исследования выявлено, что все рекомендованные препараты с доказанной эффективностью назначались реже при выписке пациентам старше 75 лет [22, 23].

Регистр IMPACT-HF проводился в медицинском центре Университета Дьюка, США, в него были включены 567 пациентов. В регистре выполнена оценка выживаемости больных с ХСН на фоне рекомендованной терапии, при которой из $\beta$-АБ использовался карведилол. Выявлено, что 60-дневная летальность и повторные госпитализации были высокими, несмотря на назначение рекомендованной терапии. И вероятно, улучшение приверженности на догоспитальном этапе сыграло бы положительную роль в уменьшении смертности данной категории больных [24, 25].

По-видимому, наиболее ценное исследование по изучению приверженности у больных с ХСН проводилось на базе QUALIFY international registry. Оценка приверженности проводилась ко всем группам препаратов следующим образом: отсутствие назначений рекомендованной терапии - 0 баллов, назначение менее $50 \%$ рекомендованной дозы - 0,5 балла, более $50 \%$ рекомендованной дозы - 1 балл - полная приверженность. Неназначение препарата в связи с противопоказанием к его приему также расценивалось как выполнение рекомендаций, т. е. приверженность врача. По результатам регистра общая приверженность врачей назначению рекомендованной терапии составила $23 \%$, средняя приверженность - $55 \%$, низкая - $22 \%$ [26].

Специфический неклинический регистр GWTG-HF (Get With The Guidelines-Heart Failure) был разработан American Heart Association's Get With The Guidelines для улучшения качества стационарного лечения и повышения приверженности терапии больных с ХCH. При исследовании был использован метод proportion of days covered (PDC) - выявлялось количество «покрытых» дней, т. е. доля дней, когда пациент принимал рекомендованный препарат. Она подсчитывается по рецептурным бланкам и таблицам, которые пациент заполняет при принятии лекарств. При PDC >80\% пациент рассматривался как приверженный. Приверженность терапии пациентов оценивалась через 90 дней и через 1 год после выписки из стационара, оценивалась она отдельно в отношении $\beta$-АБ и иАПФ. По результатам исследования приверженность пациентов была низкой. Раннее обращение после выписки в поликлинику и своевременное посещение лечебно- профилактического учреждения не были связаны с лучшей приверженностью этих пациентов. Через 1 год приверженность $\beta$-АБ была $53 \%$, иАПФ и блокаторам рецепторов ангиотензина - $48 \%$ [32].

\section{Изучение приверженности в рандомизированных контролируемых и наблюдательных \\ клинических исследованиях}

Как модель для изучения приверженности терапии особое внимание заслуживают РКИ: они позволяют получить более точные данные и практически исключают некорректные результаты. Анализ крови дает возможность точно определить концентрацию препарата, т. е. знать, принял ли пациент лекарство, рекомендованное врачом. В исследованиях с вмешательством возможно внедрять чипы в упаковки лекарственных средств, брать кровь с согласия пациента, считать рецептурные бланки, но, к сожалению, это недоступно для повседневной врачебной практики и может нести некорректную информацию.

С точки зрения оценки приверженности как таковой интересно многоцентровое рандомизированное исследование СОАСН-2. Оно должно было оценить, влияет ли на приверженность терапии место наблюдения пациента (специализированный центр пациентов c ХCH, центр первичной медицинской помощи). Оценка приверженности пациентов определялась с помощью Medication Possession Ratio (MPR) - «индекса использования препарата», который высчитывается делением количества дней приема полной дозы препарата (или выданного больному) на количество дней всего исследования (наблюдения) и выражается в процентах. Индекс MPR - важный показатель во всех РКИ, определяющих эффективность лекарственной терапии. С его помощью оценивается степень приверженности лечению. Если индекс MPR $\geq 80 \%$, можно говорить о высокой приверженности лечению, при MPR <60 \% приверженность терапии низкая. В данном исследовании пациентам выдавались рекомендованные препараты и приверженность оценивалась к отдельным их группам. Она была высокой для иАПФ и $\beta$-АБ и ниже для антагонистов минералокортикоидов $(95,2 ; 94,8 ; 87,1 \%$ соответственно). При сравнении в двух лечебно-профилактических учреждениях не выявлено статистически значимой разницы в приверженности пациентов и назначении врачами терапии согласно действующим клиническим рекомендациям [27].

В оценке приверженности часто не принимается во внимание тот факт, что к разным лекарственным препаратам у одного и того же больного может быть разная приверженность. Так, по данным нерандомизированного исследования M. Viana и соавт., пациенты с ХCH наиболее привержены терапии иАПФ, а наименее - приему петлевых диуретиков, приверженность $\beta$-АБ занимает промежуточное положение между 
обозначенными позициями. Приверженность терапии в данном исследовании оценивали с помощью Medication Event Monitoring System (MEMS). В крышку упаковки с препаратом был внедрен микрочип. Открытие упаковки приравнивалось к приему препарата. Одной из особенностей этого исследования стало то, что проводилась оценка приема каждого препарата, рекомендованного при ХСН. Ранее в аналогичных исследованиях приверженность с помощью способа MEMS определялась по отношению к одному препарату и ее переносили на все классы. Но данный способ невозможно перенести в реальную клиническую практику, и это не позволит лечащему врачу определить приверженность терапии конкретного пациента, а следовательно, и работать над ее коррекцией [33].

В РКИ НART (Heart Failure Adherence and Retention Trial) целью ставили оценку клинических исходов у двух групп, одна из которых подвергалась воздействию по улучшению приверженности терапии (с помощью обучающих программ, телефонного контроля), вторая оставалась без вмешательства. Приверженность терапии пациентов с ХСН также оценивалась по способу MEMS. В крышку от упаковки был встроен чип, который отмечал время открытия и закрытия упаковки. Эти данные обрабатывались программой дистанционно и сравнивались с фактическим назначением врача. Пациент расценивался как приверженный, если принимал $>80 \%$ рекомендованных назначений. Приверженность врача оценивалась выписыванием препаратов в соответствии с действующими на тот момент (2002 г.) рекомендациями. Выписывание препарата при наличии противопоказаний рассматривалось как неприверженность врача назначению терапии [28]. Таким образом, в этом исследовании оценивалась как приверженность врача, так и пациента - к следованию рекомендациям. Но данное РКИ также нерегистровое, пациентов включали выборочно и добровольно. По результатам HART в «группе воздействия» не уменьшилось число госпитализаций и смертность не снизилась.

В крупном международном РКИ ТОРСАТ проводилась оценка приверженности с определением концентрации одного из рекомендованных препаратов в крови - спиронолактона [34].

Широкое распространение получил метод PDC. Его использовали в РКИ, которое оценивало влияние приверженности лекарственной терапии на отдаленные исходы сердечно-сосудистых заболеваний у кардиологических больных (Нью-Йорк). В исследование были включены 4015 пациентов, и результаты показали, что большие сердечно-сосудистые события встречаются у неприверженных терапии после перенесенного инфаркта миокарда в 17,17 \%, у приверженных в $8,42 \%$ случаев $(p<0,0001)$ за 2 года [35].

В многоцентровом проспективном наблюдательном пилотном исследовании ESC-HF Pilot проводилась оценка годичной смертности амбулаторных и стационарныХ пациентов с ХСН и острой сердечной недостаточностью; оценка приверженности в цель исследования не была включена [29].

При рассмотрении нозологических форм, влияющих на приверженность, выявлено, что у больных с инфарктом миокарда в анамнезе она выше. Это может быть связано с более широким использованием программ реабилитации среди этих пациентов (на основании наблюдательного корреляционного исследования, выполненного в Сингапуре). Известно, что эти программы связаны с улучшением приверженности лечению, поскольку разъясняют цель лечения и необходимость приема каждой группы препаратов, повышая тем самым медицинскую грамотность. В упомянутом исследовании пациенты с ХСН и сопутствующей артериальной гипертензией показали лучшее соблюдение режима приема лекарств. Поскольку большинство пожилых пациентов в Сингапуре страдают артериальной гипертензией и приверженность лечению увеличивается с возрастом, это может объяснить, почему пациенты с ХСН и гипертензией имели лучшую приверженность лечению [36].

\section{Заключение}

Ознакомившись с доступной литературой, можно сказать, что достаточно хорошо изучены течение $\mathrm{XCH}$, ее исходы, назначаемая терапия, однако приверженность лечению этой когорты больных изучена недостаточно. Существующие методы оценки приверженности лечению, которые используются в клинических исследованиях, сложны для реальной практики, а опросники не адаптированы для больных. В отделе профилактической фармакотерапии ФГБУ «НМИЦ ПМ» Минздрава России создан специальный опросник для оценки фактической приверженности назначенной терапии - шкала приверженности Национального общества доказательной фармакотерапии. В связи с тем, что этот опросник не адаптирован к больным с ХCH, запланировано исследование, в ходе которого он будет адаптирован и применен, - COMPLIANCE (Assessment of adherenCe tO Medical theraPy and its influence on Long-term outcomes In pAtieNts with Chronic heart failurE In the outpatient registry). Цель исследования - оценка приверженности медикаментозной терапии и факторов, влияющих на нее, у пациентов с ХСН. Возможно, это исследование восполнит существующие пробелы в оценке приверженности у больных с ХСН в клинической практике. 
1. Мареев В.Ю., Фомин И.В., Агеев Ф.Т., и др. Клинические рекомендации ОССН-PKO-PHМOT. Сердечная недостаточность: хроническая (ХCH) и острая декомпенсированная (ОДСН). Диагностика, профилактика и лечение. Кардиология 2018;58(6S): 8-158. DOI: $10.18087 /$ cardio.2475. [Mareev V.Yu., Fomin I.V., Ageev F.T. et al. Russian Heart Failure Society, Russian Society of Cardiology. Russian Scientific Medical Society of Internal Medicine Guidelines for Heart failure: chronic (CHF) and acute decompensated(ADHF). Diagnosis, prevention and treatment. Kardiologiya $=$ Kardiologiia 2018;58(6S):8-158. (In Russ.)].

2. Van Deursen V.M., Urso R., Laroche C. et al. Co-morbidities in patients with heart failure:an analysis of the European Heart Failure Pilot Survey. Eur J Heart Fail 2014;16(1):103-11. DOI: 10.1002/ejhf.30.

3. Ефремова Е.В., Мензоров М.В., Сабитов И.А. Приверженность к лечению больных с хронической сердечной недостаточностью в условиях коморбидности. Клиническая медицина 2015;93(9):20-4. [Efremova E.V., Menzorov M.V., Sabitov I.A. Treatment compliance in patients with chronic heart failure and comorbidities. Klinicheskaya meditsina $=$ Clinical Medicine 2015;93(9):20-4. (In Russ.)].

4. Ефремова Е.В., Шутов А.М., Сабитов И.А. и др. Приверженность к лечению больных хронической сердечной недостаточностью в зависимости от функционального состояния почек. Вестник российской военно-медицинской академии 2013;(1):34-7. [Efremova E.V., Shutov A.M., Sabitov I.A. et al. Adherence to treatment in patients with chronic heart failure depending on renal function. Vestnik rossiyskoy voyenno-meditsinskoy akademii $=$ Herald of the Russian Academy of Military Medicine 2013;(1):34-7. (In Russ.)].

5. World Health Organization. Adherence to long-term therapies: evidence for action. WHO Library Cataloguing-inPublication Data. Geneva: WHO. 2003.

6. Лукина Ю.В., Дмитриева Н.А., Кутишенко Н.П. и др. Взаимосвязь и взаимовлияние аспектов безопасности лекарственного лечения и приверженности терапии у больных сердечно-сосудистыми заболеваниями (по данным амбулаторного регистра «ПРОФИЛЬ»). Кардиоваскулярная терапия и профилактика 2018;17(5):72-8. DOI: 10.15829/1728-8800-2018-5-72-78. [Lukina Yu. V., Dmitrieva N.A., Kutishenko N.P. et al. The relationship and interinfluence of aspects of therapy safety and compliance in patients with cardiovascular diseases (by the data from outpatient registry "PROFILE"). Kardiovaskulyarnaya terapiya i profilaktika $=$ Cardiovascular Therapy and Prevention 2018;17(5):72-8. (In Russ.)].

7. Лукина Ю.В., Кутишенко Н.П., Марцевич С.Ю. Проблема приверженности в современной медицине: возможности решения, влияние на результативность терапии и исходы заболевания. Рациональная Фармакотерапия в Кардиологии 2017;13(4):519-24. DOI: 10.20996/1819-6446-2017-13-4519-524. [Lukina Yu.V., Kutishenko N.P., Martsevich S.Yu. The problem of adherence to the treatment in modern medicine: possibilities of solution, impact on the effectiveness of therapy and disease outcomes. Ratsional'naya farmakoterapiya $\mathrm{v}$ kardiologii $=$ Rational Pharmacotherapy in Cardiology 2017;13(4):519-24. (In Russ.)].

8. Васюкова Н.О., Лукина Ю.В., Кутишенко Н.П. и др. Приверженность к терапии: влияет ли на нее участие пациентов в рандомизированных клинических исследованиях? Рациональная Фармакотерапия в Кардиологии 2019;15(3):386-92. DOI: 10.20996/18196446-2019-15-3-386-92. [Vasyukova N.O., Lukina Yu.V., Kutishenko N.P. et al. Medication Adherence: does Patient Participation in Randomized Clinical Trials Affect on it? Ratsional'naya Farmakoterapiya $\vee$ Kardiologii $=$ Rational Pharmacotherapy in Cardiology 2019;15(3):386-92. (In Russ.)].

9. Steinberg B.A., Shrader P., Thomas L. et al. Off-label dosing of non-vitamin K antagonist oral anticoagulants and adverse outcomes: the ORBIT-AF II registry. J Am Coll Cardiol 2016;68(24):2597-604. DOI: 10.1016/j.jacc.2016.09.966.

10. Ситникова М.Ю., Юрченко А.В., Лясникова Е.А. и др. Опыт создания и первые результаты работы Российского госпитального регистра хронической сердечной недостаточности (RUS-HFR) в трех субъектах Российской Федерации. Трансляционная медицина 2014;(1):73-81.

DOI: 10.18705/2311-4495-2014-0-1-73-81. [Sitnikova M.Yu., Yurchenko A.V., Lyasnikova E.A. et al. Experience of creation and the first results of the Russian hospital register of chronic heart failure (RUS-HFR) in three subjects of the Russian Federation. Translyatsionnaya meditsina $=$ Translational Medicine 2014;(1):73-81. (In Russ.)].

11. Виноградова Н.Г. Городской центр лечения хронической сердечной недостаточности: организация работы и эффективность лечения пациентов с хронической сердечной недостаточностью. Кардиология 2019;59(2S):31-9.
DOI: $10.18087 /$ cardio. 2621.

[Vinogradova N.G. City center for the treatment of chronic heart failure: the organization of work and the effectiveness of treatment of patients with chronic heart failure. Kardiologiya $=$ Kardiologiia 2019;59(2S):31-9. (In Russ.)].

12. Арутюнов А.Г., Драгунов Д.О., Арутюнов Г.П. и др. Первое открытое исследование синдрома острой декомпенсации сердечной недостаточности и сопутствующих заболеваний в Российской Федерации. Независимый регистр ОРАКУЛ-РФ. Кардиология 2015;55(5):512-21. DOI: 10.18565/ cardio.2015.5.12-21. [Arutyunov A.G., Dragunov D.O., Arutyunov G.P. et al. The first open study of acute decompensation syndrome of heart failure and concomitant diseases in the Russian Federation. ORACLE-RF registry. Kardiologiya = Kardiologiia 2015;55(5):12-21. (In Russ.)].

13. Терещенко С.Н., Жиров И.В., Романова Н.В. и др. Первый российский регистр больных хронической сердечной недостаточностью и фибрилляцией предсердий (РИФ-ХСН): дизайн исследования. Рациональная Фармакотерапия в Кардиологии 2015;11(6):577-81. DOI: 10.20996/1819-6446-2015-11-6577-581. [Tereshchenko S.N., Zhirov I.V., Romanova N.V. et al. The first Russian register of patients with chronic heart failure and atrial fibrillation (RIF-CHF): study design. Ratsional'naya Farmakoterapiya $v$ Kardiologii $=$ Rational Pharmacotherapy in Cardiology 2015;11(6):577-81. (In Russ.)].

14. Фомин И.В. Хроническая сердечная недостаточность в Российской Федерации: Что сегодня знаем и что должны делать. Российский кардиологический журнал 2016;8(136):7-13. DOI: 10.15829/1560-4071-2016-8-7-13. [Fomin I.V. Chronic heart failure in Russian federation: what do we know and what to do. Rossiyskiy kardiologicheskiy zhurnal $=$ Russian Journal of Cardiology 2016;8(136):7-13.

15. Арутюнов А.Г., Рылова А.К., Арутюнов Г.П. Регистр госпитализированных пациентов с декомпенсацией кровообращения (Павловский регистр). Сообщение 1. Современная клиническая характеристика пациента с декомпенсацией кровообращения. Клинические фенотипы пациентов. Журнал Сердечная недостаточность 2014;15(1):23-32. DOI: 10.18087/ rhfj.2014.1.1933. [Arutyunov A.G., Rylova A.K., Arutyunov G.P. Registry of hospitalized patients with decompensation of blood circulation (Pavlovsky register). Current clinical 
characteristics of patients with decompensation of blood circulation. Clinical phenotypes of patients. Zhurnal Serdechnaya nedostatochnost' $=$ Russian Heart Failure Journal 2014;15(1):23-32. (In Russ.)].

16. Степина Е.В., Лукьянов М.М., Бичурина М.А. и др. Назначение медикаментозной терапии, влияющей на прогноз у больных с фибрилляцией предсердий в сочетании с артериальной гипертонией, ишемической болезнью сердца, хронической сердечной недостаточностью по данным регистра РЕКВАЗА-КЛИНИКА. Кардиоваскулярная терапия и профилактика 2017;16(2):33-8. DOI: 10.15829/17288800-2017-2-33-38. [Stepina E.V., Lukyanov M.M., Bichurina M.A. et al. Prescription of medications influencing prognosis in atrial fibrillation with arterial hypertension, coronary heart disease, chronic heart failure, by the registry REKVAZA-CLINIC. Kardiovaskulyarnaya terapiya i profilaktika $=$ Cardiovascular Therapy and Prevention 2017;16(2):33-8. (In Russ.)].

17. Лукьянов М.М., Козминский А.Н., Марцевич С.Ю. и др. Больные с сочетанием хронической сердечной недостаточности, артериальной гипертонии и перенесенного ранее инфаркта миокарда: клинико-анамнестические характеристики и практика назначения ингибиторов ангиотензин-превращающего фермента, блокаторов рецепторов ангиотензина и $\beta$-адреноблокаторов, приверженность лечению (данные амбулаторного регистра РЕКВАЗА). Рациональная Фармакотерапия в Кардиологии 2017;13(2):207-12. DOI: 10.20996/1819-6446-2017-13-2207-212. [Loukianov M.M., Kozminsky A.N., Martsevich S.Yu. et al. Patients with combination of chronic heart failure, hypertension and history of myocardial infarction: clinical and anamnestic characteristics, administration of ace inhibitors, angiotensin receptor blockers, $\beta$-blockers and adherence to the drug therapy (data of outpatient registry RECVASA). Ratsional'naya Farmakoterapiya $v$ Kardiologii $=$ Rational Pharmacotherapy in Cardiology 2017;13(2):207-12. (In Russ.)].

18. Штегман О.А., Поликарпов Л.С., Новиков О.М. Приверженность к лечению амбулаторных больных хронической сердечной недостаточностью. Сибирский медицинский журнал 2013;28(2):78-82. [Shtegman O.A., Polikarpov L.S., Novikov O.M. Adherence to treatment in outpatients with chronic heart failure. Sibirskiy meditsinskiy zhurnal $=$ The Siberian Medical Journal 2013;28(2):78-82. (In Russ.)].

19. Митрофанова И.С., Коц Я.И., Вдовенко Л.Г. Новые пути повышения эффек- тивности лечения больных хронической сердечной недостаточностью. Журнал Сердечная недостаточность 2016;9(4):164-6. [Mitrofanova I.S., Koc Ya.I., Vdovenko L.G. New ways to improve the effectiveness of treatment of patients with chronic heart failure. Zhurnal Serdechnaya nedostatochnost' = Russian Heart Failure Journal 2016;9(4):164-6. (In Russ.)].

20. Adams K.F.Jr., Fonarow G.C., Emerman C.L. et al. Characteristics and outcomes of patients hospitalized for heart failure in the United Stated: rationale, design, and preliminary observations from the first 100,000 cases in the Acute Decompensated Heart Failure National Survey (ADHERE). Am Heart J 2005;149(2):209-16. DOI: 10.1016/j.ahj.2004.08.005.

21. Yancy C.W., Lopatin M., Stevenson L.W. et al. Clinical presentation, management, and in-hospital outcomes of patients admitted with acute decompensated heart failure with preserved systolic function: a report from the Acute Decompensated Heart Failure National Registry (ADHERE) database. J Am Coll Cardiol 2006;47(1):76-84.

DOI: $10.1016 /$ j.jacc.2005.09.022.

22. Fonarow G.C., Abraham W.T., Albert N.M. et al. OPTIMIZE-HF Investigators and Hospitals. Age- and gender-related differences in quality of care and outcomes of patients hospitalized with heart failure (from OPTIMIZE-HF). Am J Cardiol 2009;104(1):107-15.

DOI: 10.1016/j.amjcard.2009.02.057.

23. Fonarow G.C., Abraham W.T., Albert N.M. et al. Influence of a performance-improvement initiative on quality of care for patients hospitalized with heart failure: results of the Organized Program to Initiate Lifesaving Treatment in Hospitalized Patients With Heart Failure (OPTIMIZE-HF) Arch Intern Med 2007;167(14):1493-502. DOI: 10.1001/archinte.167.14.1493.

24. Patel K., Fonarow G.C., Ekundayo O.J. et al. Beta-blockers in older patienfts with heart failure and preserved ejection fraction: class, dosage, and outcomes. Int J Cardiol 2014;173(3):393-401. DOI: 10.1016/j.ijcard.2014.03.005.

25. O'Connor C.M., Stough W.G., Gallup D.S. et al. Demographics, clinical characteristics, and outcomes of patients hospitalized for decompensated heart failure: observations from the IMPACTHF registry. J Card Fail 2005;11(3):200-5. DOI: 10.1016/j.cardfail.2004.08.160.

26. Komajda M., Schöpe J. Wagenpfeil S. et al. Physicians' guideline adherence is associated with long-term heart failure mortality in outpatients with heart failure with reduced ejection fraction: the QUALIFY international registry.
Eur J Heart Fail 2019;21(7):921-9. DOI: 10.1002/ejhf.1459.

27. Luttik M.L., Jaarsma T., van Geel P.P. et al. Long-term follow-up in optimally treated and stable heart failure patients: primary care vs. heart failure clinic. Results of the COACH-2 study. Eur J Heart Fail 2014;16(11):1241-8. DOI: 10.1002/ejhf.173.

28. Calvin J.E., Shanbhag S., Avery E. et al. Adherence to evidence-based guidelines for heart failure in physicians and their patients: lessons from the Heart Failure Adherence Retention Trial (HART). Congest Heart Fail 2012;18(2):73-8. DOI: 10.1111/j.1751-7133.2011.00263.x.

29. Maggioni A.P., Dahlström U., Filippatos G. et al. EURObservational Research Programme: regional differences and 1-year follow-up results of the Heart Failure Pilot Survey (ESC-HF Pilot). Eur J Heart Fail 2013;15(7):808-17. DOI: $10.1093 /$ eurjhf/hft050.

30. Shoaib A., Farag M., Nolan J. et al. Mode of presentation and mortality amongst patients hospitalized with heart failure? A report from the First Euro Heart Failure Survey. Clin Res Cardiol 2019;108:510-9. DOI: $10.1007 / \mathrm{s} 00392-018-1380-6$.

31. Pulignano G., Del Sindaco D., Tavazzi L. et al. Clinical features and outcomes of elderly outpatients with heart failure followed up in hospital cardiology units: data from a large nationwide cardiology database (IN-CHF Registry). Am Heart J 2002;143(1):45-55. DOI: $10.1067 / \mathrm{mhj} .2002 .119608$.

32. Chang L.L., Xu H., de Vore A.D. et al. Timing of Postdischarge Follow-Up and Medication Adherence Among Patients With Heart Failure. J Am Heart Assoc 2018;7(7):007998. DOI: 10.1161/JAHA.117.007998.

33. Viana M., Laszczynska O., Mendes S. et al. Medication adherence to specific drug classes in chronic heart failure. J Manag Care Spec Pharm 2014;20(10):1018-26. DOI: 10.18553/jmcp.2014.20.10.1018.

34. Pitt B., Pfeffer M.A., Assmann S.F. et al. Spironolactone for heart failure with preserved ejection fraction. N Engl J Med 2014;370(15):1383-92. DOI: 10.1056/NEJMoa1313731.

35. Bansilal S., Castellano J.M., Garrido E. et al. Assessing the impact of medication adherence on long-term cardiovascular outcomes. J Am Coll Cardiol 2016;68(8):789-801. DOI: $10.1016 /$ j.jacc.2016.06.005

36. Ling R.Z.Q., Jiao N., Hassan N.B. et al. Adherence to diet and medication and the associated factors among patient with chronic heart failure in a multi-ethnic society. Heart Lung 2020;49(2):144-50. DOI: 10.1016/j.hrtlng.2019.11.003. 


\section{ORCID авторов/ORCID of authors}

Э.Т. Гусейнова/Е.Т. Guseinova: https://orcid.org/0000-0003-3908-5497

Н.П. Кутишенко/N.P. Kutishenko: https://orcid.org/0000-0001-6395-2584

Ю.В. Лукина/Yu.V. Lukina: https://orcid.org/0000-0001-8252-3099

C.Н. Толпыгина/S.N. Tolpygina: https://orcid.org/0000-0003-0160-0158

В.П. Воронина/V.P. Voronina: https://orcid.org/0000-0001-5603-7038

C.Ю. Марцевич/S.Yu. Martsevich: https://orcid.org/0000-0002-7717-4362

Конфликт интересов. Авторы заявляют об отсутствии конфликта интересов.

Conflict of interest. The authors declare no conflict of interest.

Финансирование. Работа выполнена без спонсорской поддержки.

Financing. The work was performed without external funding. 